\title{
Quantum Hall effect in gapped graphene heterojunctions
}

\author{
J. L. Lado, ${ }^{1,2}$ J. W. González, ${ }^{1}$ and J. Fernández-Rossier ${ }^{1, *}$ \\ ${ }^{1}$ International Iberian Nanotechnology Laboratory - INL, Av. Mestre José Veiga, 4715-330 Braga, Portugal \\ ${ }^{2}$ Departamento de Física Aplicada, Universidad de Santiago, Santiago de Compostela, Spain
}

(Received 17 April 2013; published 31 July 2013)

\begin{abstract}
We model the quantum Hall effect in heterostructures made of two gapped graphene stripes with different gaps, $\Delta_{1}$ and $\Delta_{2}$. We consider two main situations, $\Delta_{1}=0, \Delta_{2} \neq 0$, and $\Delta_{1}=-\Delta_{2}$. They are different in a fundamental aspect: only the latter features kink states that, when intervalley coupling is absent, are protected against backscattering. We compute the two-terminal conductance of heterostructures with channel length up to $430 \mathrm{~nm}$, in two transport configurations, parallel and perpendicular to the interface. By studying the effect of disorder on the transport along the boundary, we quantify the robustness of kink states with respect to backscattering. Transport perpendicular to the boundary shows how interface states open a backscattering channel for the conducting edge states, spoiling the perfect conductance quantization featured by the homogeneously gapped graphene Hall bars. Our results can be relevant for the study of graphene deposited on hexagonal boron-nitride, as well as to model graphene with an interaction-driven gapped phase with two equivalent phases separated by a domain wall.
\end{abstract}

DOI: 10.1103/PhysRevB.88.035448

PACS number(s): 73.43.-f, 73.22.Pr, 72.80.Vp

\section{INTRODUCTION}

The Hall conductance in a quantum Hall bar is so accurately described by $\sigma_{x y}=n \frac{e^{2}}{h}$, where $n$ is an integer number, that it is used ${ }^{1}$ as our standard definition of the ratio of such fundamental constants as the square of the electron charge $e^{2}$ and the Planck constant $h$. The origin of this extraordinary quantization, by which the conduction properties of a device are independent of the material properties, is intimately linked to the fact that in quantum Hall bars transport takes place only through the edges which host chiral states for which backscattering is forbidden. ${ }^{2,3}$ In turn, the existence of chiral edge states that permit nondissipative transport is warranted by the topological order of the electronic states of the two-dimensional gas states. For two decades, this state of affairs was observed at cryogenic temperatures under high applied magnetic fields, in two-dimensional electron gases, hosted by carefully designed modulation-doped semiconductor heterostructures. The discovery of a quantum Hall effect on graphene, ${ }^{4,5}$ even at room temperature ${ }^{6}$ on one side and the proposal $^{7-9}$ and subsequent discovery ${ }^{10}$ of quantum spin Hall insulators on the other, has dramatically expanded the materials and experimental conditions under which nondissipative quantum transport linked to topological order can occur.

Most of the striking electronic properties of graphene are related to the absence of a gap separating the conduction and valence bands, which can thereby be described in terms of massless Dirac fermions. ${ }^{11,12}$ In particular, the magnetoelectronic properties of graphene are fundamentally different from a nonrelativistic two-dimensional electron gas on three counts: ${ }^{13}$ the existence of two identical sets of Landau levels, for electrons and holes, the scaling of their energy with $\sqrt{B}$, as opposed to linear scaling of nonrelativistic fermions, and the existence of the $n=0$ Landau level with zero energy. These properties make the quantum Hall effect in graphene ${ }^{4,5}$ different from the one originally discovered in GaAs twodimensional electron gases. ${ }^{1}$

There are several physical scenarios that motivate the study of the electronic properties of gapped graphene: First, a gap could be opened by interaction-driven electronic order, ${ }^{14-16}$ especially when a high magnetic field is applied, and second, as a result of the influence of substrates such as $\mathrm{SiC}$ (Refs. 17 and 18) or hexagonal boron nitride (BN), ${ }^{19}$ although the lattice mismatch is known to complicate this second possibility. ${ }^{20-23}$ Third, BN itself can be described with the tight-binding Hamiltonian of gapped graphene and the $\vec{k} \cdot \vec{p}$ Hamiltonian of other two-dimensional materials with hexagonal symmetry, such as $\mathrm{MoS}_{2}$, can be described with a massive Dirac Hamiltonian. ${ }^{2-26}$ Fourth, intrinsic spin orbit coupling also opens a gap in graphene, ${ }^{7,8}$ with different signs at the two valleys, albeit very small. ${ }^{27}$

All of this leads to the question of how magnetotransport properties of graphene and graphenelike materials change when a gap opens or, in the long wavelength limit, how massive and massless Dirac fermions are different in their reaction to an applied magnetic field. It turns out that when the gap is opened by a constant staggered potential, i.e., a potential that acts with opposite sign in the two sublattices of graphene, as it happens with pseudospin magnetism or in the case of hexagonal $\mathrm{BN}$, the answer to the question is quite straightforward from a theoretical standpoint. This occurs because there is a simple one-to-one relation between the energy levels and wave functions of a bipartite lattice Hamiltonian with no staggered potential and those of the same lattice when a constant staggered potential is added. ${ }^{28,29}$ This relation permits us to anticipate that the quantum Hall effect of massive Dirac fermions is much closer to that of massless Dirac fermions than to that of nonrelativistic electrons. ${ }^{30}$

The situation becomes more interesting when the gap, or mass, is not homogeneous. This could be the case, for instance, of a heterostructure made of two graphene or graphenelike materials with different gaps $\Delta_{1}$ and $\Delta_{2}$, such as the atomic layers of hybridized BN and graphene domains, ${ }^{31,32}$ or if the gap is substrate driven and, due to lattice mismatch, features amplitude modulations larger than the graphene unit cell. ${ }^{22,23}$ Here we study the electronic properties of heterojunctions formed between two gapped graphenelike systems, with 
different gaps, in the quantum Hall regime. When decoupled, both Hall bars would have their own set of chiral edge states. When coupled, the way edge states merge determines the electronic properties of the interface, which is the focus of this work.

As we discuss below, we encounter two types of interface states. The hybridization of pre-existing edge states that carry electrons in opposite directions will give rise to interface states that can carry electrons in both directions. In contrast, the merger of two bars with opposite gaps gives rise to two interface states whose energy lies within the gap. At zero magnetic field these states can be rationalized as follows. When restricted to one valley $\tau$, graphene electrons can be assigned a Chern number $\tau \frac{|\Delta|}{2 \Delta}$, with $\tau= \pm 1$. According to the index theorem, ${ }^{33}$ the interface between two insulators with Chern numbers $n_{1}$ and $n_{2}$ should host $\mathcal{Q}=\left|n_{1}-n_{2}\right|$ domain wall states, which will correspond to zero modes in the case of one dimension ${ }^{34}$ and chiral bands or kink states in two dimensions. These are very similar to the recently discussed kink states in the interface of two graphene bilayers ${ }^{35-37}$ and multilayers ${ }^{38}$ with a gap opened by the application of an electric field with opposite direction at the two sides of the junction.

The rest of this paper is organized as follows. In Sec. II we review the electronic structure of gapped graphene under the influence of a perpendicular magnetic field, both within the conventional tight-binding model as well as its long wavelength counterpart, the massive Dirac fermions. In Sec. III we study the electronic structure of graphene heterojunctions in a geometry that preserves translational invariance along one direction, which simplifies the discussion and permits the appearance of interface states to be unveiled. In Sec. IV we study the quantum transport properties of the kink states in these structures, including the effect of disorder. In Sec. V we consider heterojunctions formed by two semi-infinite Hall bars made of gapped graphenelike systems with different gaps. Transport in this type of heterojunction could be used to probe the interface states, which enables backscattering between the otherwise-decoupled chiral edges states. In Sec. VI we summarize our conclusions.

\section{STRIPES OF GAPPED GRAPHENE IN A MAGNETIC FIELD}

\section{A. Tight-binding Hamiltonian}

Here we review the well-studied tight-binding model for graphene under the influence of a perpendicular magnetic field. ${ }^{12,13,39,40}$ We consider the standard tight-binding model for graphene, with one orbital per atom and first-neighbor hopping $t \approx 2.7 \mathrm{eV}$, with a staggered potential $\Delta(\vec{R})$ that might be position dependent. A given graphene unit cell, located at $\vec{R}$, has two atoms, denoted by $A$ and $B$. Using this notation, the zero field tight-binding Hamiltonian reads

$$
H=\sum_{\vec{R}}\left(a_{\vec{R}}^{\dagger}, b_{\vec{R}}^{\dagger}\right)\left(\begin{array}{cc}
\Delta(\vec{R}) \delta_{\vec{R}, \vec{R}^{\prime}} & t_{\vec{R}, \vec{R}^{\prime}} \\
t_{\vec{R}, \vec{R}^{\prime}} & -\Delta(\vec{R}) \delta_{\vec{R}, \vec{R}^{\prime}}
\end{array}\right)\left(\begin{array}{l}
a_{\vec{R}^{\prime}} \\
b_{\vec{R}^{\prime}}
\end{array}\right),
$$

where $t_{\vec{R}, \vec{R}^{\prime}}$ is nonzero only for first neighbors and $a_{\vec{R}}, b_{\vec{R}}$ annihilate an electron at the $A$ and $B$ sites of unit cell $\vec{R}$ defined on a honeycomb lattice. Taking $\Delta$ as a constant along the entire system, this Hamiltonian describes graphene with a gap of $2 \Delta$ in both valleys. In the rest of this paper the spin degree of freedom is ignored. Results for noninteracting electrons with spin can be obtained by adding the Zeeman shift to the obtained bands.

Within this tight-binding description, the effect of the applied magnetic field is included by replacing the hopping $t_{1,2}$ between sites 1 and 2 of the lattice of the $B=0$ Hamiltonian by $t_{1,2} \rightarrow t_{1,2} e^{i \Phi_{1,2}}$, where ${ }^{39-41}$

$$
\Phi_{1,2}=\frac{e}{\hbar} \int_{1}^{2} \vec{A} \cdot d \vec{r}
$$

is the circulation of the vector potential $\vec{A}$ associated to the magnetic field $\vec{B}$, and the labels 1 and 2 stand for the coordinates of the two atoms whose hopping integral is being calculated. This is the lattice analogous of the canonical substitution for the free electrons, where the momentum operator $\vec{p}$ is replaced by $\vec{p}-e \vec{A}$. Notice that the phase $\Phi_{1,2}$ that modulates the hopping is proportional to the ratio of the magnetic flux per unit cell and the magnetic flux quantum $\Phi_{0}=\frac{h}{e}$.

In the following we assume that graphene lies in the $z=0$ plane, and we take $\vec{B}=B(0,0,1)$. Taking advantage of the gauge symmetry, we choose

$$
\vec{A}=B(-y, 0,0)
$$

so that

$$
\Phi_{1,2}=-\frac{e B}{\hbar} \frac{\left(x_{2}-x_{1}\right)\left(y_{2}+y_{1}\right)}{2},
$$

where $\left(x_{i}, y_{i}\right)$ are the Cartesian coordinates to atoms 1 and 2. With this choice, the Hamiltonian keeps translational invariance along the $x$ direction.

\section{B. Effective mass approximation}

Whereas the tight-binding approach provides a fairly complete description of the noninteracting electrons in graphene under the effect of a magnetic field, as we discuss below, most of the results for states with energies in the neighborhood of the Dirac points can be rationalized by making use of the $\vec{k} \cdot \vec{p}$ description of the bands in the continuum limit. ${ }^{42}$ The magnetic field introduces a new length scale in the problem:

$$
l_{B}=\sqrt{\frac{\hbar}{e B}} .
$$

We assume a sufficiently high magnetic field so that $l_{B}<W$, where $\mathrm{W}$ is the width of the ribbon, and hence the bulk quantum states become localized, the spectrum of states away from the edges becomes discrete, the bulk is an insulator, and dispersive and conducting states are only possible at edges. For typical magnetic fields, we also have $a \ll l_{B}$, where $a=\sqrt{3} a_{C C}$ is the graphene lattice constant, which enables a description of the energy levels in terms of an effective $\vec{k} \cdot \vec{p}$ Hamiltonian.

The effective $\vec{k} \cdot \vec{p}$ or effective mass Hamiltonian turns out to be isomorphic to the Dirac Hamiltonian at the two valleys, ${ }^{11}$

$$
H_{\tau}=v_{F}\left(\Pi_{x} \sigma_{x}+\tau \Pi_{y} \sigma_{y}\right)+\Delta \sigma_{z},
$$

where $\vec{\Pi} \equiv \vec{p}-e \vec{A}$ is the canonical momentum operator, $v_{F}=3 t a_{C C} / 2 \hbar, \vec{\sigma}$ are the Pauli matrices describing the 
graphene sublattice degree of freedom, and $\tau= \pm 1$ describes the valley index.

Using the gauge defined in Eq. (3) leads to

$$
H_{\tau}=\left(\begin{array}{cc}
\Delta & v_{F}\left[p_{x}-e B y+i \tau p_{y}\right] \\
v_{F}\left[p_{x}-e B y-i \tau p_{y}\right] & -\Delta
\end{array}\right) .
$$

This Hamiltonian is translationally invariant along the $x$ direction, so that we can assume its eigenfunctions are products $e^{i k_{x} x} \vec{\phi}_{n}\left(k_{x}, y\right)$ which permit replacing the operator $p_{x}$ by the quantum number $\hbar k_{x}$ in Eq. (7). We define the dimensionless canonical operators:

$$
Q\left(k_{x}\right) \equiv\left(\frac{y}{l_{B}}-k_{x} l_{B}\right)
$$

and

$$
P \equiv \frac{l_{B}}{\hbar} p_{y},
$$

combined with the intrinsic energy scale associated with the Fermi velocity,

$$
\frac{\hbar \omega_{0}}{2} \equiv \frac{\hbar v_{F}}{l_{B}} .
$$

Notice that the role of $k_{x}$ is to shift the eigenvalues of the $Q\left(k_{x}\right)$ operator. It is very convenient to define the ladder operators:

$$
\alpha\left(k_{x}\right)=\frac{1}{\sqrt{2}}\left(Q\left(k_{x}\right)+i P\right),
$$

which satisfy $\left[\alpha\left(k_{x}\right), \alpha\left(k_{x}\right)^{\dagger}\right]=1$. For simplicity, in the following we omit the $k_{x}$ dependence of the $\alpha$ operator. We thus can write the Hamiltonian for the $\tau=+1$ valley as

$$
H_{+}\left(k_{x}, p_{y}\right)=\left(\begin{array}{cc}
\Delta & \frac{-\hbar \omega_{0}}{\sqrt{2}} \alpha^{\dagger} \\
\frac{-\hbar \omega_{0}}{\sqrt{2}} \alpha & -\Delta
\end{array}\right),
$$

whereas for the $\tau=-1$ valley the Hamiltonian reads $H_{-}\left(k_{x}, p_{y}\right)=H\left(k_{x}, p_{y}\right)^{\dagger}$.

In order to find the eigenfunctions and eigenvalues of these Hamiltonians, it is convenient to compute their square:

$$
H_{\tau= \pm 1}^{2}=\left(\begin{array}{cc}
\mathcal{H}^{2}(\tau) & 0 \\
0 & \mathcal{H}^{2}(-\tau)
\end{array}\right),
$$

where $\mathcal{H}^{2}(\tau) \equiv \Delta^{2}+\frac{1}{2}\left(\hbar \omega_{0}\right)^{2}\left(\alpha^{\dagger} \alpha+\frac{1-\tau}{2}\right)$.

In the following we denote the eigenstates of the operator $\alpha^{\dagger} \alpha$ as $\phi_{n}$, with eigenvalues $n=0,1, \ldots$ and eigenfunctions $\phi_{n}$. The states $\phi_{n}$ are the standard harmonic oscillator wave functions, centered around $y=k_{x} l_{B}^{2}$. The eigenstates of $H_{\tau= \pm 1}^{2}$, and thereby eigenstates of $\mathcal{H}_{\tau}$, denoted by $\vec{\phi}_{n}$, fall in two categories: the so-called zero Landau level, with a sublattice polarized wave function, and the normal Landau levels.

\section{Landau levels}

From Eq. (13) it can be seen right away that eigenstates can be written as

$$
\vec{\phi}_{n}(\tau)=\left(\begin{array}{c}
A_{n} \phi_{n} \\
B_{n} \phi_{n-\tau}
\end{array}\right)
$$

where $A_{n}$ and $B_{n}$ are coefficients that are determined by requesting that $\vec{\phi}_{n}$ are also eigenstates of the Dirac equation. The corresponding eigenenergies are $E_{n}^{2}=\Delta^{2}+$ $\frac{1}{2}\left(\hbar \omega_{0}\right)^{2}\left(n+\frac{1-\tau}{2}\right)$. Therefore the general equation for the eigenvalues of the Dirac Hamiltonian under the influence of a perpendicular two-dimensional field are $\mathrm{a}^{30,43}$

$$
E_{n}(\tau)= \pm \sqrt{\Delta^{2}+\frac{1}{2}\left(\hbar \omega_{0}\right)^{2}\left(n+\frac{1-\tau}{2}\right)}
$$

It is apparent that the Landau level energies are independent of $k_{x}$. Therefore they give rise to flatbands with a very large degeneracy. Moreover, there is an additional twofold valley degeneracy given by

$$
E_{n}(\tau=-1)=E_{n+1}(\tau=+1) .
$$

Of course, Eq. (14) is only mathematically defined if both $n$ and $n+\tau$ are non-negative. As a result, the minimal value that $n$ can take is $n=1$ for $\tau=-1$, and $n=0$ for $\tau=+1$. For these states, the energy can be written as $E_{N}= \pm \sqrt{\Delta^{2}+\frac{1}{2}\left(\hbar \omega_{0}\right)^{2}(N+1)}$, where $N+1$ is a strictly positive integer. In summary, these states come in doublets, on account of the valley degree of freedom, and in addition have electron-hole symmetry.

\section{Zero Landau level}

In addition to these states, for each valley there is an extra eigenstate of $H^{2}$ with the minimal eigenvalue $H_{\tau}^{2} \vec{z}_{\tau}=\Delta^{2} \vec{z}_{\tau}$. They are

$$
\vec{z}_{\tau=+1}=\left(\begin{array}{c}
\phi_{0} \\
0
\end{array}\right)
$$

and

$$
\vec{z}_{\tau=-1}=\left(\begin{array}{c}
0 \\
\phi_{0}
\end{array}\right)
$$

We thus see that these wave functions are very special: they are sublattice polarized. It can be verified right away that these wave functions satisfy

$$
H_{\tau} \vec{z}_{\tau}=\tau \Delta \vec{z}_{\tau}
$$

Thus the energy of the zeroth Landau levels becomes valley dependent due to the mass term $\Delta$, as shown in Fig. 1(a). We can relate this to the fact that the mass introduces an orbital magnetic moment with valley-dependent orientation. ${ }^{44}$

A summary of the energy spectrum for gapped graphene electrons under the influence of a perpendicular magnetic field, described within the $\vec{k} \cdot \vec{p}$ approximation, is shown in Fig. 1 . Whereas for all the finite $|n|$ levels each valley contributes with one Landau level, so that they come in couples in graphene, the $n=0$ Landau levels are valley polarized so that there is only one for the electron sector and one for the hole sector. For $\Delta=0$ these two $n=0$ levels are degenerate. However, this degeneracy is lifted for gapped graphene and a gap between them is open. This discussion has ignored the spin degree of freedom, which would add an additional twofold degeneracy to all the levels broken by the Zeeman splitting. 


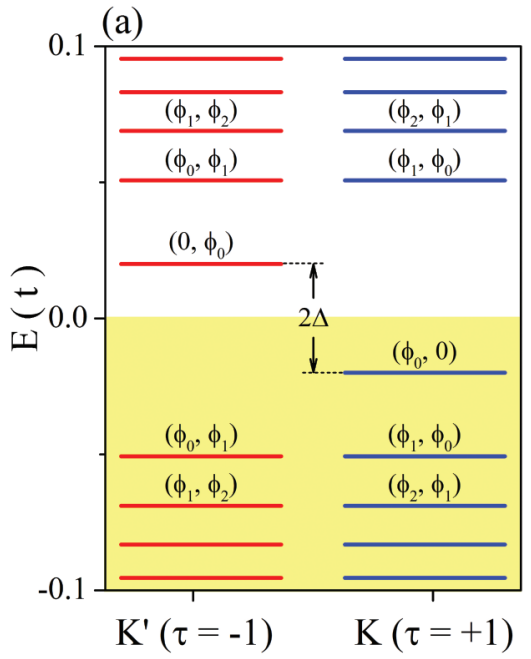

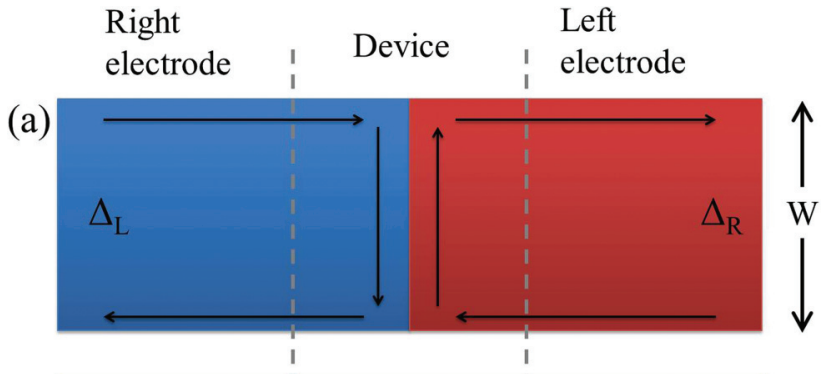

(b)

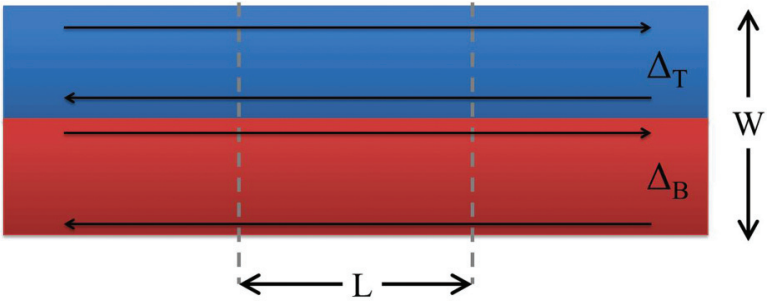

(c)
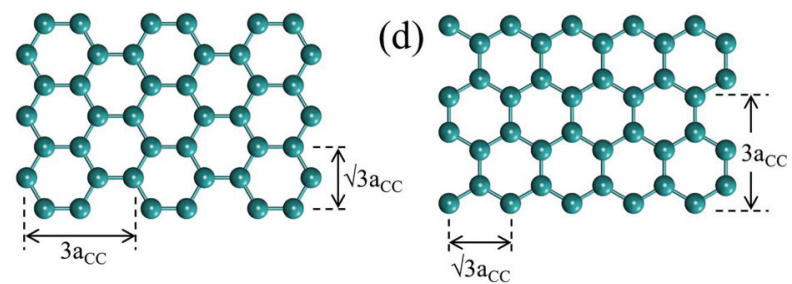

FIG. 2. (Color online) Scheme of the two geometries considered in the text: (a) perpendicular transport to the interface between the two different gap regions and (b) for the parallel transport. Arrows indicate edge, interface, and kink states in the quantum Hall regime. (c) Detail of the armchair ribbon $N_{W}=7(W=0.74 \mathrm{~nm})$ and $N_{L}=3$ $(L=1.3 \mathrm{~nm})$ and (d) zigzag ribbon edges $N_{W}=4(W=0.9 \mathrm{~nm})$ and $N_{L}=5(L=1.2 \mathrm{~nm})$.
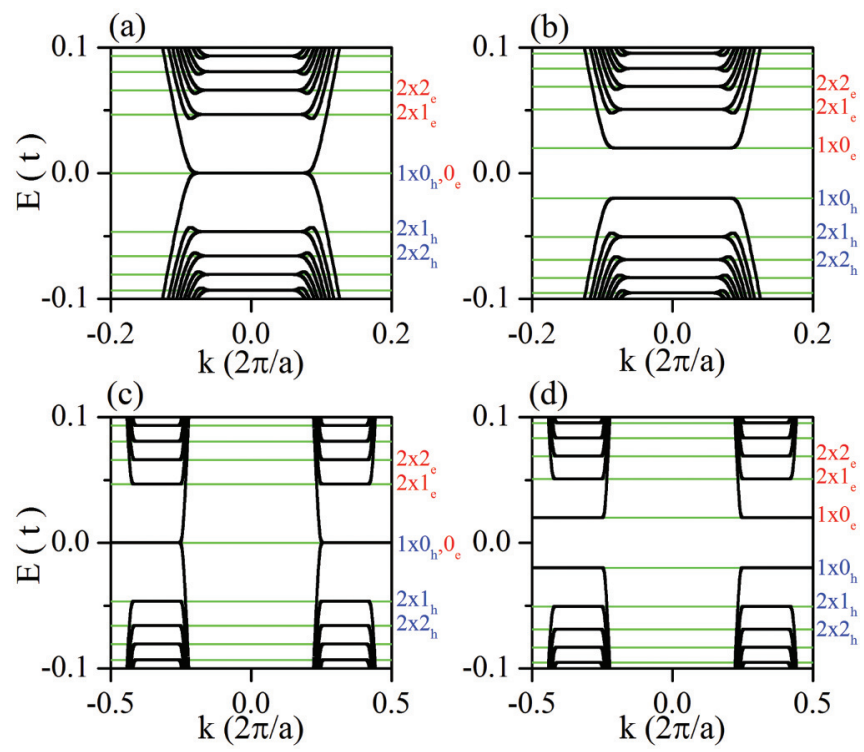

FIG. 3. (Color online) Band structure in the quantum Hall regime for uniform massless armchair (a) and zigzag (c) nanoribbons, and of the massive $(\Delta=0.02 \mathrm{t})$ ribbon armchair (b) and zigzag (c). In the four cases the magnetic field is fixed $\Phi=0.0002(B=15.8 \mathrm{~T})$ and the dimensions are $N_{W}=1000(W=123 \mathrm{~nm}$ for armchair $W=$ $213 \mathrm{~nm}$ for the zigzag). Green lines correspond to the analytical eigenvalues in Eq. (15). 
The dispersive states correspond to states localized at the edges. There is a linear relation between localization along the transverse direction of the ribbon and the momentum $k_{x}$, as expected from Eq. (8). The edge velocity $v_{n}(k)=\frac{1}{\hbar} \frac{\partial \epsilon_{n}(k)}{\partial k}$ changes from one edge to the other. The emergence of these chiral edge states whose energy lies in the gap between Landau levels anticipates the very peculiar quantized transport properties of the system, characteristic of the quantum Hall effect. $^{3}$

In agreement with the effective mass results, the flatbands (Landau levels) have a twofold degeneracy, both in the armchair and zigzag cases, except for the $n=0$ level. In the case of armchair termination, the degeneracy occurs at the same $k$ point, whereas in the case of zigzag, there are two sets of bands that can be ascribed to the two valleys. ${ }^{45}$ The $\Delta$ term shifts the position of all the Landau levels and splits the $n=0$ levels, opening a transport gap even at the edges, also in agreement with the effective mass results. The presence of two flatbands, at a given valley, associated with the $n=0$ Landau is in apparent conflict with the effective mass approximation (see Fig. 1). It turns out that one of the two $n=0$ flatbands at each valley is an edge state rather than a bulk state. ${ }^{45}$ This statement is further clarified in the next section.

\section{ELECTRONIC PROPERTIES OF GRAPHENE QUANTUM HALL BARS WITH INHOMOGENEOUS $\Delta$}

We are now in position to study the electronic states of graphene quantum Hall bars where $\Delta$ is not constant. For that matter, we consider the simplest situation, a ribbon of width $W$ where top and bottom halves have a different mass $\Delta_{T}$ and $\Delta_{B}$. We consider two cases $\Delta_{T}=-\Delta_{B}$ and $\Delta_{T} \neq 0, \Delta_{B}=0$, both armchair and zigzag terminations (four cases in total). The band structures, together with the velocity density,

$$
j_{x}(y, E) \equiv \sum_{k_{x}, n}\left|\phi_{n, k_{x}}(y)\right|^{2} \frac{\partial \epsilon_{n}\left(k_{x}\right)}{\partial k_{x}} \delta\left[E-\epsilon_{n}\left(k_{x}\right)\right],
$$

are shown in Fig. 4.

For a given Landau level $n$ with wave function $\psi_{n}(k, y)$, and within a given valley, there is a relation between the $k$ quantum number and the average vertical position $\left\langle\psi_{n}(k, y)|y| \psi_{n}(k, y)\right\rangle$. Thus plots of the velocity density as a function of $y$ provide complementary information to bands $\epsilon_{n}(k)$. In panels (a) and (b) we show the armchair ribbon with $\Delta_{T} \neq 0, \Delta_{B}=0$. It is apparent that, according to their location in the ribbon, we can distinguish three types of states: edge states, bulk states, and, in contrast with ribbons with homogeneous $\Delta$, interface states located at the boundary between the massive and massless sectors. For the bulk states we obtain two different sets of flat Landau levels, corresponding to the massless and massive halves, respectively. The edge states are quite similar to those of the homogeneous mass case. For the $n \neq 0$ Landau levels, the interface states can be interpreted as the hybridization of the two pairs of counterpropagating edge states from the homogeneous $\Delta$ sectors. This hybridization results in two intertwined oscillating bands. For $n=0$, Landau levels of the interface states are unique and join the two pairs of $n=0$ Landau levels. They can also be interpreted as regular edge
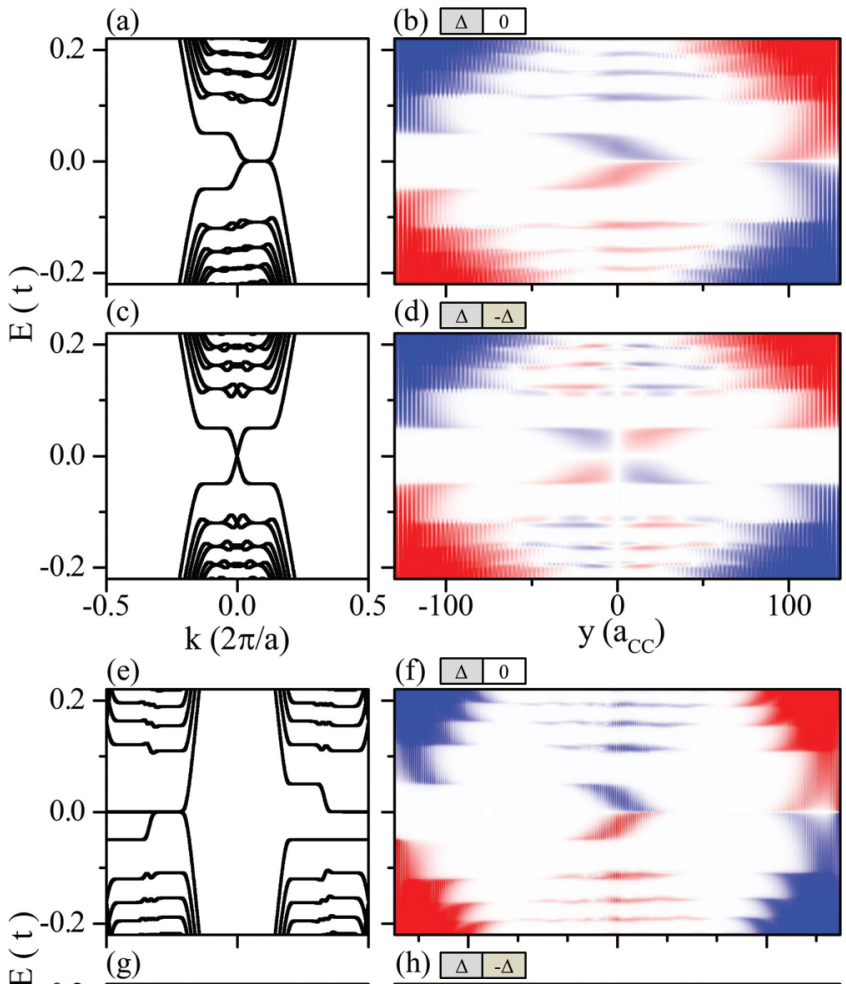

(d) \begin{tabular}{|l|l|}
\hline$\Delta$ & $-\Delta$ \\
\hline
\end{tabular}
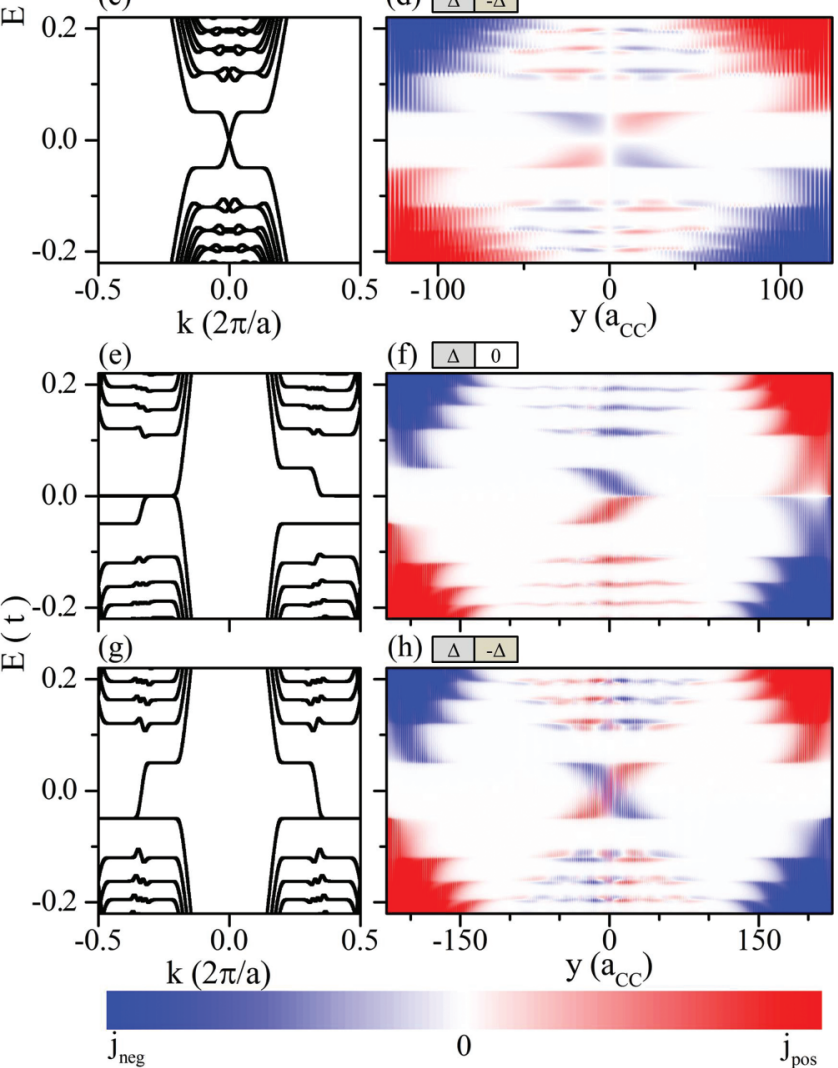

FIG. 4. (Color online) Band structure (left row) and the corresponding velocity density defined by Eq. (20) (right row). Here we consider transport parallel to the interface [see Fig. 2(b)] for armchair (a)-(d) and (e)-(h) zigzag ribbons. We fix the mass of the upper half of the ribbon $\Delta_{T}=0.05 \mathrm{t}$, and the lower half of the ribbon has either $\Delta_{B}=0$ [(a), (b) and (e), (f)] or $\Delta_{B}=-\Delta_{T}$ [panels (c), (d) and (g), (h)]. The magnetic flux is $\Phi=1.1 \times 10^{-3}(B=87 \mathrm{~T})$ and the width of the ribbon is $N_{W}=300(W=36.9 \mathrm{~nm}$ for armchair $W=63.9$ $\mathrm{nm}$ for zigzag).

states of the massless half confined by the gap on one side and the vacuum on the other.

Results become more interesting for the armchair ribbon with $\Delta_{T}=-\Delta_{B} \neq 0$, shown in Figs. 4(c) and 4(d). Bulk Landau levels, edge states, and $n \neq 0$ interface states are very similar to the previous case. The main difference occurs for the interface states for the $n=0$ Landau levels, which fill the gap almost completely. The two counterpropagating interface states undergo a small anticrossing at zero energy. These interface states that reside in the gap are quite similar to the kink states reported for bilayer graphene with a positiondependent, off-plane electric field. ${ }^{35-38,47}$ 
The discussion for zigzag ribbons goes along the same line. For a zigzag ribbon with $\Delta_{T} \neq 0, \Delta_{B}=0$ [Figs. 4(c) and 4(f)], we have two replicas of the Landau levels and their edge or interface states for each valley. For the $n \neq 0$ Landau levels there are bulk flatbands, and dispersive edge and interface states, very much like in the case of armchair ribbon. For the $n=0$ Landau levels, Fig. 4 shows four types of bulk states, attending to the sublattice $\left(\sigma_{z}\right)$ and valley ( $\tau_{z}$ ) indexes: (i) gapless with $\left(\sigma_{z}=+1, \tau_{z}=+1\right)$, (ii) gapless with $\left(\sigma_{z}=-1, \tau_{z}=-1\right)$, both with zero energy, (iii) and (iv) gapped $(\Delta>0)$, with energy $\tau_{z} \Delta$ and either $\left(\sigma_{z}=\right.$ $\left.+1, \tau_{z}=+1\right)$ or $\left(\sigma_{z}=-1, \tau_{z}=-1\right)$, respectively, as expected from the effective mass theory. In addition, at each valley there is a kink state that joins the gapless $n=0$ Landau level with the corresponding gapped state. This kink state shares a spectral range with the edge states.

The properties of the zigzag ribbon with $\Delta_{T}=-\Delta_{B}$ are in line with the other cases. The main feature here is the presence of a kink state at each valley that, in contrast with the armchair ribbon, has no anticrossings and covers the gap completely. The wave functions of the kink states are located at the interface, as expected. The velocities of the kink states are opposite for the valley. This is one of the main results of this manuscript: we predict the existence of counterpropagating valley polarized states at the interface of two graphene quantum Hall bars with opposite masses.

We have also studied the electronic structure of ribbons for which the variation of $\Delta$ is not abrupt (not shown in the figures). For that matter, we have chosen a model with a central region of width $L_{m t}$ where $\Delta$ changes linearly [see Fig. 7(d)]. As long as $L_{m t}$ is smaller than the $l_{B}$, the bands for this system are qualitatively the same than those shown in Fig. 4.

\section{TRANSPORT PROPERTIES OF KINK STATES}

We now discuss the robustness with respect to disorder of transport properties of the kink states found in ribbons with $\Delta_{T}=-\Delta_{B}$. For that matter, we consider the geometry shown in Fig. 2, an infinite stripe with three regions: two semi-infinite electrodes without disorder joined by a central region, of length $L$, that features an Anderson disorder potential: ${ }^{48}$

$$
V=\sum_{i} V_{i} c_{i}^{\dagger} c_{i},
$$

where $V_{i}$ is a random variable uniformly distributed over the interval $\left[-V_{0}, V_{0}\right]$, where the energy scale $V_{0}$ sets the strength of the disorder potential. The transmission is calculated for each disordered configuration and averaged over different disorder configuration realizations.

Making use of the partition method and the Green's function approach, ${ }^{49}$ outlined in Appendix 1, we compute the scattering transmission function $T(E)$, which relates to the two-terminal elastic conductance through the Landauer formula $G=$ $\frac{e^{2}}{h} T\left(E_{F}\right)$, as mentioned in Eq. (A6). The transmission function is the sum over the transmission coefficients $T_{n}$ of the channels $n$ available at a given energy. For an ideal transmission channel without backscattering, $T_{n}=1$. A completely blocked channel gives $T_{n}=0$.

The edge states in quantum Hall bars are the canonical example of ideally transmitting channels, with $T_{n}=1$. This (a) (b)

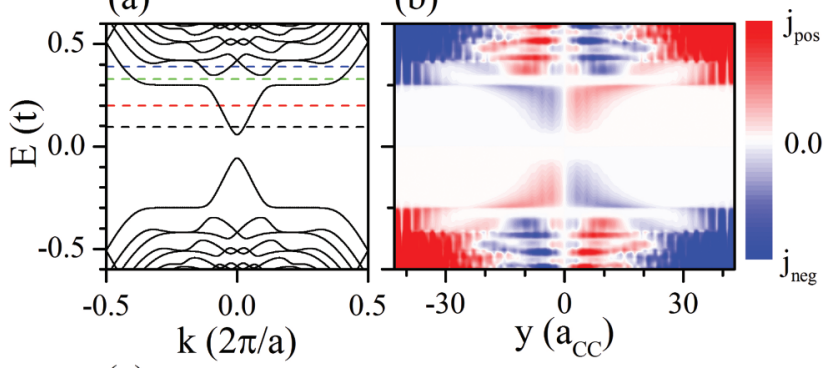

(c)
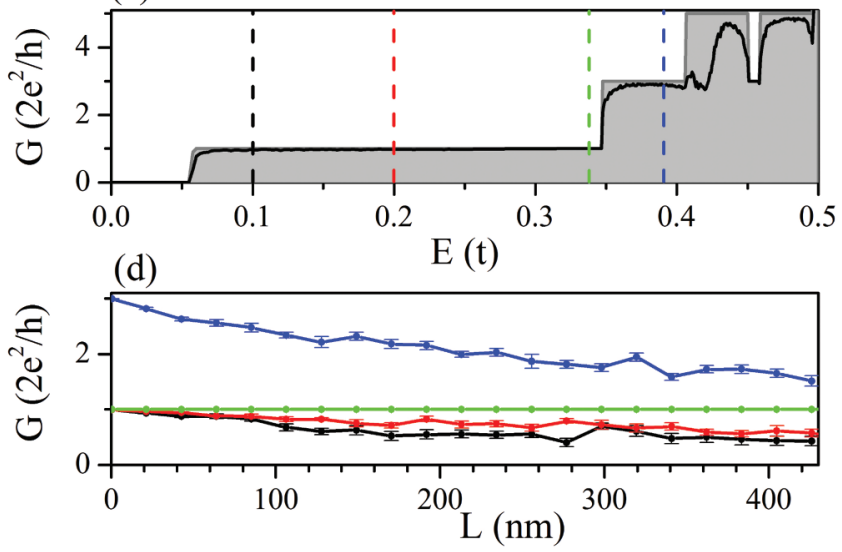

FIG. 5. (Color online) Electronic properties of an armchair ribbon with a stepwise constant gap $\Delta_{T}=-\Delta_{B}=0.3 \mathrm{t}$ [see Fig. 2(b)] under a magnetic field $\Phi=0.0081(B=640 \mathrm{~T}), N_{W}=100(W=$ $12.3 \mathrm{~nm}$ ). (a) Energy bands, (b) velocity density map for the disorderfree structure, (c) two-terminal conductance as a function of $E_{F}$ (in units of the hopping t) with disorder (black line). As a reference the conductance of the disorder-free ribbon has been included as a shadow region. (d) Two-terminal conductance as a function of channel length for four different energies [marked in (a) and (c)]. In the disorder cases (c), (d) the Anderson parameter is $V_{0}=0.1 \mathrm{t}$, an average over ten disorder configurations was performed, and the error bars reflect the standard deviation.

leads to a quantized two-terminal conductance, $G=n \frac{2 e^{2}}{h}$, where $n$ is an integer number. The computed two-terminal conductance is actually related to the Hall conductance as measured in a four-terminal quantum Hall bar. ${ }^{50}$

We consider first the transport properties of the armchair ribbon with opposite mass $\Delta_{T}=-\Delta_{B}$. Since the calculation of the transmission coefficients requires the determination of the Green's function of a system with $2 \times N_{W} \times N_{L}$ atoms, and we consider lengths $N_{L}$ up to several thousands, it is computationally convenient to choose a smaller $N_{W}$ but large enough so that there is no interedge coupling. This also makes it necessary to take unrealistically large values of $B$. However, we expect that the simulated structures have the same properties than wider ribbons with smaller magnetic fields.

We study now the transmission, as a function of energy, for a fixed length of the disordered region $N_{L}$, shown in Fig. 5(c). The shadow background with quantized steps shows the transmission without disorder, and the black line is the transmission for a length $N_{L}=30$ for $V_{0}=0.1 \mathrm{t}$. The stepwise function for $V_{0}=0$, i.e., in the absence of disorder, reflects 
the number of modes at a given energy, starting from 0 when the energy lies inside the gap (coming from anticrossing of the kink states) and increasing as the Fermi energy reaches new edge states.

The effect of disorder in Fig. 5(c) for a fixed channel length is clearly energy dependent. This is more clearly seen in Fig. 5(d), where we plot the conductance, averaged over ten disorder configurations, at the four energies marked in Figs. 5(a) and 5(c), as a function of the length of the ribbon. The vertical error bars reflect the standard deviation. It is apparent that as the length of the transport channel $L$ increases, the transmission tends to the quantized values when the energy crosses edge states (green line) but tends to zero in the case of the kink states (black and red lines). Furthermore, when the energy crosses both edge and interphase states (blue line), the channels corresponding to the interphase states attenuate as the length is increased. Therefore backscattering is possible, which is expected since two states with opposite velocities coexist in the interface region.

When the same analysis is done for a zigzag ribbon with $\Delta_{T}=-\Delta_{B}$, the results found are quite similar (see Fig. 6). Therefore our transport results show that, unlike edge states, kink states living in the interface of two gapped graphene regions with opposite gaps are not protected. It must be stressed that, very much like in the case of gapped bilayer graphene, the existence of two valleys prevents the robustness of kink states with respect to disorder, as it would happen in the case of kink states located at the domain wall for a two-dimensional electron gas of Dirac electrons.

(a)

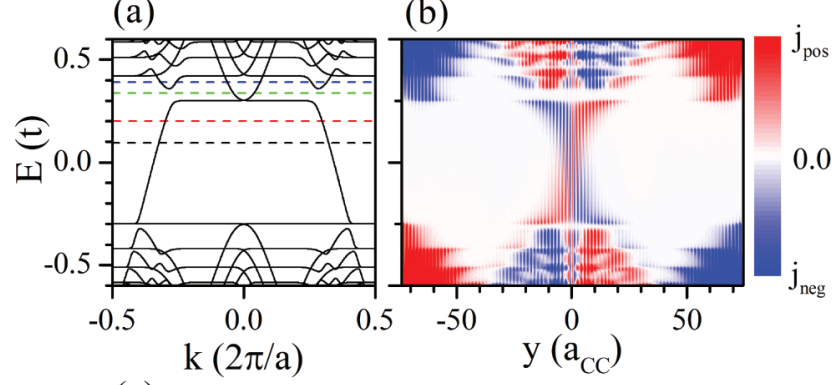

(c)

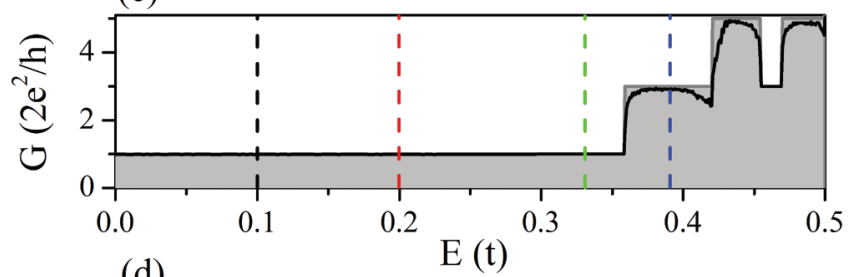

(d)

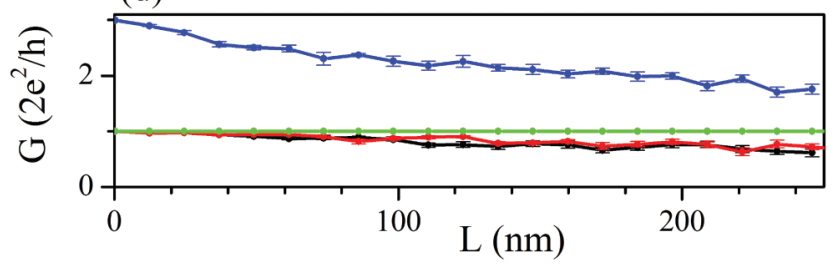

FIG. 6. (Color online) (a)-(d) as in Fig. 5 for the case of a zigzag ribbon with a stepwise constant gap $\Delta$ with $\Delta_{T}=-\Delta_{B}=0.3 \mathrm{t}$, under a magnetic field with $\Phi=0.0081(B=640 \mathrm{~T}), N_{W}=100$ $(W=21.3 \mathrm{~nm})$.

\section{QUANTUM HALL TRANSPORT PROPERTIES OF GAPPED GRAPHENE HETEROJUNCTIONS}

In this section we study transport across the boundary that separates two gapped graphene quantum Hall bars. Our main goal is to analyze the backscattering that takes place at the interface due to interface states that connect the edges. A scheme of this heterostructure, made of two regions with constant mass $\Delta_{L}=-\Delta_{R}=\Delta$ that meet at an abrupt interface, is shown in Fig. 2(a). We consider transport across the interface region which, in addition to the stepwise constant mass has Anderson disorder with $V_{0}=0.1$ t over a distance $N_{L}=30$. Away from the interface each side of the junction hosts a set of Landau levels and edge states as described in Sec. II. Because both electrodes have a gap $2 \Delta$, conductance is only possible for states with energy $E>\Delta$.

Both sides of the structure host chiral edge states that do not backscatter, even in the presence of disorder, unless electrons at one edge can undergo scattering to the other edge. This could be enabled if interface states, i.e., states running along the interface perpendicular to the transport direction, are available at the Fermi energy and are not completely blocked by disorder. Below we show that interface states, perpendicular to the transport direction, can act as an efficient shortcut between the right-goers in one edge and the left-goers in the other (see Fig. 2), providing a backscattering channel which destroys the conductance quantization. It must be stressed that, in this geometry, the in-gap kink states studied in the previous section do not play a role in transport due to the absence of transport states in the electrodes inside the gap.

In Figs. 7 and 8 we show results for the conductance of both armchair and zigzag heterojunctions. The interface between the two armchair (zigzag) ribbons with opposite mass is given by a zigzag (armchair) boundary whose properties can be related to those of the infinite graphene zigzag (armchair) ribbon with inhomogeneous mass. For that matter, the top panel of Figs. 7 and 8 shows the velocity density map obtained for the infinite ribbon with $\Delta_{T}=-\Delta_{B}$. Whereas a priori this velocity density should not be identical to the boundary of Fig. 2(a), due to the finite width of the Hall bar, our results indicate that it allows the existence of backscattering induced by interface states to be anticipated.

Since we are interested in the interface states as a source of backscattering, it is not necessary to consider a long central region with disorder, as we did in the previous section. In addition, the role of disorder here could be to reduce the efficiency of the interface states to produce backscattering, thereby improving the conductance.

For both armchair and zigzag geometries the conductance is zero for $E<\Delta=0.3 \mathrm{t}$ and is quantized in $G=\frac{2 e^{2}}{h}$ above the gap, over an energy interval that coincides with the absence of interface states, shown in panel (a) of Figs. 7 and 8 . The comparison of the velocity density for an infinite ribbon with the two-terminal conductance for both heterostructures reveals a relation between the existence of interface states in the junction [(a) panels] and the backscattering in transport [(b) panels]. In particular, the two spectral regions with null velocity density at the interface give quantized plateaus of conductance. This is particularly apparent in the 

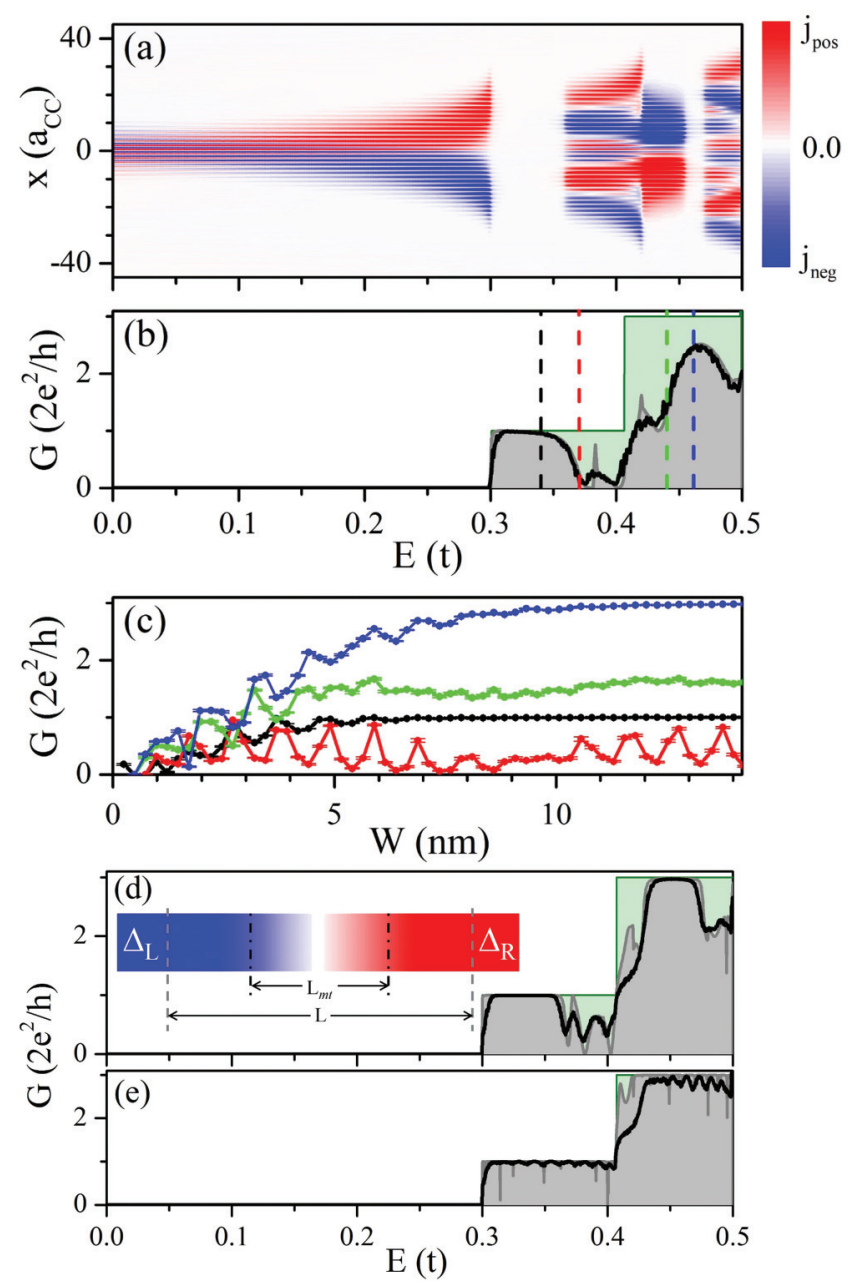

FIG. 7. (Color online) Electronic properties of a graphene heterostructure made of two semi-infinite armchair ribbons with opposite mass $\Delta_{L}=-\Delta_{R}=0.3 \mathrm{t}$ [see Fig. 2(a)], under a magnetic field $\Phi=0.0081(B=640 \mathrm{~T})$. (a) Velocity density map corresponding to the zigzag infinite ribbon along the interface. (b) Two-terminal conductance as a function of $E_{F}$ with disorder (black line) for a central region of length $N_{L}=30(L=12.8 \mathrm{~nm})$ and width $N_{W}=50$ ( $W=6.1 \mathrm{~nm}$ ), and an average over ten disorder configurations was performed. As reference we include the conductance of the disorderfree ribbon as a gray line for $\Delta_{L}=-\Delta_{R}$ and the homogeneous mass $\Delta_{L}=\Delta_{R}$ as a green shadow. (c) Two-terminal conductance as a function of the ribbon width for four different energies [marked in (b)] calculated with disorder and a channel length $N_{L}=30$, averaged over 100 configurations. All the disordered cases have been calculated with Anderson disorder $V_{0}=0.1 \mathrm{t}$. (d) Conductance as a function of $E_{F}$, with a linear mass transition from $\Delta_{L}$ to $\Delta_{R}$, for a channel length $L=21.3 \mathrm{~nm}$ and a region of lineal gap transition $L_{m t}=1.7 \mathrm{~nm}$ in (a) and for $L_{m t}=8.5 \mathrm{~nm}$ in (d). The color scheme is the same as that in (b).

case of the plateau with $G=\frac{2 e^{2}}{h}$ for $E$ right above the band gap.

The connection between interface states and backscattering is further confirmed by studying the conductance as a function of the width of the Hall bar at four different energies. In Figs. 7(c) and 8(c) at the black and blue energies, the backscattering is completely canceled for sufficiently wide
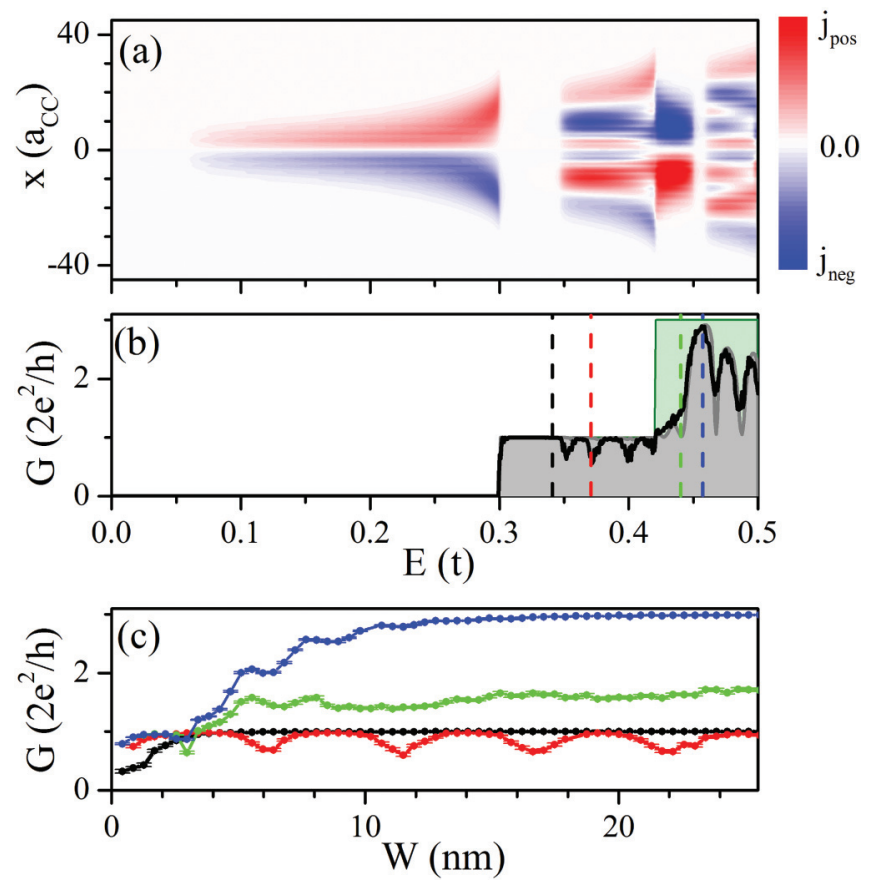

FIG. 8. (Color online) (a) Velocity density map corresponding to the armchair infinite ribbon (along the interface, see text). (b), (c) As in Fig. 7, for the case of zigzag ribbons with opposite mass $\Delta_{L}=-\Delta_{R}=0.3 \mathrm{t}$, under a magnetic field with $\Phi=0.0081$ $(B=640 \mathrm{~T})$.

ribbons. At those energies, there are no interface states. In contrast, for red and green energies, the conductance oscillates as a function of the ribbon width. This can be interpreted as follows. As the ribbon width is increased, the discrete spectrum of interface states shifts. When an interface state is in resonance with the electrode states, the backscattering is possible and conductance is reduced. In contrast, peaks in the transmission correspond to poor matching between the incoming state and the interface state. It is apparent that the amplitude of the oscillations does not decrease significantly as the width of the ribbon increases, even in the presence of disorder. This suggest that the localization length of the interface states along the direction perpendicular to transport is longer than the ribbon width. The results are qualitatively similar in the case of a heterostructure made of zigzag ribbon with opposite masses. In this case the domain wall separating the two regions with opposite mass runs along the armchair direction.

Disorder seems to have two effects on the interface-stateinduced backscattering in these heterojunctions. On one hand, it is probably increasing the mixing of edge states to interface states, which should enhance the backscattering. On the other hand, for sufficiently wide ribbons disorder could result in localization of the interface states that are responsible for backscattering, which should decrease the backscattering. The comparison of the two curves with and without disorder in the middle panels of Figs. 7 and 8 indicate that the dominance of one effect over the other depends on energy. In general, the interface-induced backscattering effect is not qualitatively affected by disorder. 


\section{A. Smooth gap transition}

We now briefly discuss the effect on the previous results of a nonabrupt change of the $\Delta$ across the junction. For that matter, we consider transport across a region where the gap changes linearly in the direction of the ribbon. We assume a device with a fixed total length $L$, which contains a central region determined by $L_{m t}$, where the gap changes from $\Delta_{L}$ in the left electrode to $\Delta_{R}=-\Delta_{L}$ at the right, as depicted in the inset of Fig. 7(d). Anderson disorder is present in the entire device of length $L$. For brevity we limit our discussion to the case of armchair ribbons, although we have also obtained similar results for the zigzag case.

In Figs. 7(d) and 7(e) we present the two-terminal conductance as a function of energy for two different values of the length scale $L_{m t}$ that characterize the soft mass transition, $L_{m t}=1.7$ and $L_{m t}=8.5 \mathrm{~nm}$ [or in units of the magnetic length defined in Eq. (5), $L_{m t}=0.6 l_{B}$ and $L_{m t}=3 l_{B}$ ]. It is apparent that for the sharper transition $\left(L_{m t}=0.6 l_{B}\right)$ the curves $G(E)$ [Fig. 7(d)] are very similar to the abrupt transition shown in Fig. 7(b). For the softer mass transition $\left(L_{m t}=3 l_{B}\right)$, shown in Fig. 7(e), the backscattering induced at the interface is depleted.

\section{DISCUSSION AND CONCLUSIONS}

We have studied the electronic properties of graphene quantum Hall bars with a position-dependent mass $\Delta$. We have considered the case of stepwise constant $\Delta$. We have found that at the boundary of two regions with opposite $\Delta$, both in-gap kink states and interface states appear. Interface states arise from the mixing of counterpropagating edge states that coexist in energy. In contrast, kink states arise in the domain wall between two gapped regions and do not coexist in energy with bulk states. In the case of zigzag ribbons, there is one kink state at each valley, whose propagation direction changes from valley to valley.

We have studied transport in two different configurations that would allow either the in-gap kink states or the interface states to be probed. The study of transport parallel to the interface between two graphene regions with opposite $\Delta$ in the quantum Hall regime permits one to study the properties of the kink states (Sec. III). We have found that the coexistence in real space of two counterpropagating kink states, corresponding to the two valleys, leaves them unprotected from backscattering created by disorder. In the case of zigzag ribbon, backscattering requires changing valleys, which in turn requires short-range scattering, provided by the Anderson disorder.

The study of transport across the junction of two semiinfinite ribbons with opposite mass, discussed in Sec. V, permits studying the effect of interface states as sources of backscattering. Our calculations show how an incoming electron to the junction from a chiral edge state could scatter to a kink state propagating from one edge to the opposite, enabling backscattering at the specific energies at which interface states exist.

Our calculations represent a toy model for situations in which graphene quantum Hall bars have a position-dependent mass. This could be the case of a mass driven by electronic order, for which different ground states could coexist in the sample, or a mass modulated by the interaction with a substrate with a very large commensuration period. ${ }^{22,51}$

\section{ACKNOWLEDGMENTS}

This work has been financially supported by MEC-Spain (Grants No. FIS2010-21883-C02-01 and No. CONSOLIDER CSD2007-0010, and Generalitat Valenciana through Grants No. ACCOMP/2012/127 and No. PROMETEO/2012/011). J.L.L. would like to gratefully acknowledge the INL for their hospitality and summer scholarship program.

\section{APPENDIX: CALCULATION OF TRANSMISSION}

\section{Partition method and Green's functions}

In this Appendix we give the technical details of the calculation of the transmission function $T(E)$, which yields the two-terminal conductance through the Landauer formula. ${ }^{49}$ The calculation method applies for a one-dimensional system that can be split in three regions: a central "device" of finite size described with the Hamiltonian matrix $H_{c}$ coupled to two semi-infinite electrodes, left and right leads, described by $H_{L}$ and $H_{R}$. In matrix form the Hamiltonian reads

$$
H=\left(\begin{array}{cc}
H_{C} & V \\
V^{\dagger} & H_{S}
\end{array}\right),
$$

where

$$
H_{S}=\left(\begin{array}{cc}
H_{L} & 0 \\
0 & H_{R}
\end{array}\right)
$$

and

$$
V=\left(V_{L} V_{R}\right),
$$

with $V_{L}$ and $V_{R}$ the coupling to the left and right leads, which we assume to be otherwise decoupled from each other. A central quantity in the method is the Green's function $\hat{G}(E \hat{I}-$ $\hat{H})=\hat{I}$, where $\hat{I}$ is the identity matrix.

The projection of the Green function operator over the central region can be written, after some algebra, as

$$
G_{C}=\left[E \hat{I}-H_{C}-\Sigma_{R}-\Sigma_{L}\right]^{-1},
$$

where the self-energies $\Sigma_{\eta}$ of the $\eta=L, R$ lead are given by

$$
\Sigma_{\eta}=V_{\eta} g_{\eta} V_{\eta}^{\dagger}
$$

and $g_{\eta}=\left(E \hat{I}_{\eta}-\hat{H}_{\eta}\right)^{-1}$ are the projections of the Green's function operators over the $\eta=L, R$ spaces.

The conductance can be calculated in the linear response regime within the Landauer formalism as a function of the energy $E$. In terms of the Green's function of the system, ${ }^{49}$ it reads

$$
G=\frac{2 e^{2}}{h} T(E)=\frac{2 e^{2}}{h} \operatorname{Tr}\left[\Gamma_{L} G_{C} \Gamma_{R} G_{C}^{\dagger}\right],
$$

where $T(E)$ is the transmission function across the conductor, and $\Gamma_{\eta}=i\left[\Sigma_{\eta}-\Sigma_{\eta}^{\dagger}\right]$ is the coupling between the conductor and the $\eta=L, R$ lead.

\section{Determination of the electrode Green's function}

Equations (A4)-(A6) are all expressed in terms of the electrode Green's function $g_{\eta}$. In particular, when represented 
in a local basis, it is the so-called surface term of the $g_{\eta}$ matrices which is needed. When the electrode Hamiltonian is written in the form

$$
H_{\eta}=\left(\begin{array}{ccccc}
h_{\eta} & v_{\eta} & 0 & \ldots & \\
v_{\eta}^{\dagger} & h_{\eta} & v_{\eta} & 0 & \\
0 & v_{\eta}^{\dagger} & \ddots & \ddots & \\
\vdots & 0 & \ddots & \ddots & \ddots
\end{array}\right),
$$

it can be shown that the electrode Green's function satisfies the self-consistent equation

$$
g_{\eta}=\left(E-h_{\eta}-v_{\eta} g_{\eta} v_{\eta}^{\dagger}\right)^{-1}
$$

and corresponds to the central and one of the most timeconsuming steps in the calculation. We label the electrode Green's function obtained in step $(i)$ of the iteration procedure as $g_{\eta}^{i}$. We have found that the stability of the self-consistent procedure is improved by using the following algorithm to compute step $(i)$, for $i \geqslant 1$,

$$
g_{\eta}^{i}=\alpha g_{\eta}^{i-1}+\beta g_{\eta}^{i-2}+(1-\alpha-\beta) g_{\eta}^{i-3},
$$

with the the initial guess $g_{\eta}^{0}=g_{\eta}^{-1}=g_{\eta}^{-2}=g_{\eta}^{-3}$ and $\alpha, \beta$ are mixing parameters.

\section{Calculation of the transmission}

The other source of computational overhead in the calculation of Eq. (A6) is the inversion of the central region $H_{C}$ matrix, renormalized with the self-energies, to obtain $G_{C}$. However, this can be greatly simplified by taking advantage of two facts. First, in the computation of the transmission, only a few matrix elements of the device Green's function are actually needed, in particular, those involved in the $\Gamma_{\eta} G_{C}$ products, which are a minor fraction given the surface nature of the $\Gamma$ matrices. Second, the device Hamiltonian can be written as a tridiagonal block matrix. This permits us to use specific techniques for tridiagonal matrices that make the procedure much faster. ${ }^{52}$ Taking advantage of this approach, it is possible to compute the transmission of 300-nm-long bars in a desktop computer.
* On leave from Departamento de Física Aplicada, Universidad de Alicante, Spain.

${ }^{1}$ K. Von Klitzing, Rev. Mod. Phys. 58, 519 (1986).

${ }^{2}$ R. B. Laughlin, Phys. Rev. B 23, 5632 (1981).

${ }^{3}$ B. I. Halperin, Phys. Rev. B 25, 2185 (1982).

${ }^{4}$ K. S. Novoselov, A. K. Geim, S. V. Morozov, D. Jiang, M. I. Katsnelson, I. V. Grigorieva, S. V. Dubonos, and A. A. Firsov, Nature (London) 438, 197 (2005).

${ }^{5}$ Y. Zhang, Y.-W. Tan, H. L. Stormer, and P. Kim, Nature (London) 438, 201 (2005).

${ }^{6}$ K. S. Novoselov, Z. Jiang, Y. Zhang, S. V. Morozov, H. L. Stormer, U. Zeitler, J. C. Maan, G. S. Boebinger, P. Kim, and A. K. Geim, Science 315, 1379 (2007).

${ }^{7}$ C. L. Kane and E. J. Mele, Phys. Rev. Lett. 95, 226801 (2005).

${ }^{8}$ C. L. Kane and E. J. Mele, Phys. Rev. Lett. 95, 146802 (2005).

${ }^{9}$ B. Bernevig, T. Hughes, and S. C. Zhang, Science 314, 1757 (2006).

${ }^{10}$ M. Konig, S. Wiedmann, C. Brüne, A. Roth, H. Buhmann, L. W. Molenkamp, X. L. Qi, and S. C. Zhang, Science 318, 766 (2007).

${ }^{11}$ G. W. Semenoff, Phys. Rev. Lett 53, 2449 (1984).

${ }^{12}$ A. H. Castro Neto, F. Guinea, N. M. R. Peres, K. S. Novoselov, and A. K. Geim, Rev. Mod. Phys. 81, 109 (2009).

${ }^{13}$ V. P. Gusynin and S. G. Sharapov, Phys. Rev. Lett. 95, 146801 (2005).

${ }^{14}$ H. Min, G. Borghi, M. Polini, and A. H. MacDonald, Phys. Rev. B 77, 041407(R) (2008).

${ }^{15}$ Y. Araki, Phys. Rev. B 84, 113402 (2011).

${ }^{16}$ G. W. Semenoff, Phys. Scr. 2012, 014016 (2012).

${ }^{17}$ S. Y. Zhou, G.-H. Gweon, A. V. Fedorov, P. N. First, W. A. de Heer, D.-H. Lee, F. Guinea, A. H. Castro Neto, and A. Lanzara, Nat. Mater. 6, 770 (2007).

${ }^{18}$ A. Bostwick, T. Ohta, J. L. McChesney, K. V. Emtsev, T. Seyller, K. Horn, and E. Rotenberg, New J. Phys. 9, 385 (2007).

${ }^{19}$ G. Giovannetti, P. A. Khomyakov, G. Brocks, P. J. Kelly, and J. van den Brink, Phys. Rev. B 76, 073103 (2007).
${ }^{20}$ C. R. Dean, A. F. Young, I. Meric, C. Lee, L. Wang, S. Sorgenfrei, K. Watanabe, T. Taniguchi, P. Kim, K. L. Shepard, and J. Hone, Nat. Nanotechnol. 5, 722 (2010).

${ }^{21}$ J. Xue, J. Sanchez-Yamagishi, D. Bulmash, P. Jacquod, A. Deshpande, K. Watanabe, T. Taniguchi, P. Jarillo-Herrero, and B. J. LeRoy, Nat. Mater. 10, 282 (2011).

${ }^{22}$ M. Kindermann, B. Uchoa, and D. L. Miller, Phys. Rev. B 86, 115415 (2012)

${ }^{23}$ B. Hunt, J. D. Sanchez-Yamagishi, A. F. Young, K. Watanabe, T. Taniguchi, P. Moon, M. Koshino, P. Jarillo-Herrero, and R. C. Ashoori, Science 340, 1427 (2013).

${ }^{24}$ D. Xiao, G. B. Liu, W. Feng, X. Xu, and W. Yao, Phys. Rev. Lett. 108, 196802 (2012).

${ }^{25}$ X. Li, F. Zhang, and Q. Niu, Phys. Rev. Lett. 110, 066803 (2013).

${ }^{26}$ A. Kormanyos, V. Zolyomi, N. D. Drummond, P. Rakyta, G. Burkard, and V. Fal'ko, arXiv:1304.4084.

${ }^{27}$ Y. Yao, F. Ye, X. L. Qi, S. C. Zhang, and Z. Fang, Phys. Rev. B 75, 041401(R) (2007).

${ }^{28}$ V. M. Pereira, J. M. B. Lopes dos Santos, and A. H. Castro Neto, Phys. Rev. B 77, 115109 (2008).

${ }^{29}$ D. Soriano and J. Fernández-Rossier, Phys. Rev. B 85, 195433 (2012).

${ }^{30}$ P. M. Krstajic and P. VasilopoulosPhys. Rev. B 86, 115432 (2012).

${ }^{31}$ L. Ci, L. Song, C. Jin, D. Jariwala, D. Wu, Y. Li, A. Srivastava, Z. F. Wang, K. Storr, L. Balicas, F. Liu, and P. M. Ajayan, Nat. Mater. 9, 430 (2010).

${ }^{32}$ J. Jung, Z. Qiao, Q. Niu, and A. H. MacDonald, Nano Lett. 12, 2936 (2012).

${ }^{33} \mathrm{G}$. E. Volovik, The Universe in a Helium Droplet (Oxford University Press, New York, 2003).

${ }^{34}$ R. Jackiw and C. Rebbi, Phys. Rev. D 13, 3398 (1976).

${ }^{35}$ I. Martin, Ya. M. Blanter, and A. F. Morpurgo, Phys. Rev. Lett. 100, 036804 (2008).

${ }^{36}$ J. Li, I. Martin, M. Buttiker, and A. F. Morpurgo, Nat. Phys. 7, 38 (2011). 
${ }^{37}$ P. Vargas, Appl. Phys. Lett. 98, 262107 (2011).

${ }^{38}$ J. Jung, F. Zhang, Z. Qiao, and A. H. MacDonald, Phys. Rev. B 84, 075418 (2011).

${ }^{39}$ K. Wakabayashi and M. Sigrist, Phys. Rev. Lett. 84, 3390 (2000).

${ }^{40}$ K. Wakabayashi, Phys. Rev. B 64, 125428 (2001).

${ }^{41}$ R. Peierls, Z. Phys. 80, 763 (1933).

${ }^{42}$ J. M. Luttinger and W. Kohn, Phys. Rev. 97, 869 (1955).

${ }^{43}$ M. Koshino and T. Ando, Phys. Rev. B 81, 195431 (2010).

${ }^{44}$ D. Xiao, W. Yao, and Q. Niu, Phys. Rev. Lett. 99, 236809 (2007).

${ }^{45}$ L. Brey and H. A. Fertig, Phys. Rev. B 73, 195408 (2006).

${ }^{46}$ K. Nakada, M. Fujita, G. Dresselhaus, and M. S. Dresselhaus, Phys. Rev. B 54, 17954 (1996).
${ }^{47}$ Z. Qiao, J. Jung, Q. Niu, and A. H. MacDonald, Nano Lett. 11, 3453 (2011).

${ }^{48}$ J. M. Ziman, Models of Disorder: The Theoretical Physics of Homogeneously Disordered Systems (Cambridge University Press, Cambridge, UK, 1979).

${ }^{49}$ S. Datta, Electronic Transport in Mesoscopic Systems (Cambridge University Press, Cambridge, UK, 1995).

${ }^{50}$ D. J. Thouless, Topological Quantum Numbers in Nonrelativistic Physics (World Scientific, Singapore, 1998).

${ }^{51}$ E. J. Mele, Phys. Rev. B 84, 235439 (2011).

${ }^{52}$ D. Petersen, H. B. Sorensen, P. Hansen, S. Skelboe, and Kurt Stokbro, J. Comput. Phys. 227, 3174 (2008). 